\title{
Effect of wheat flour with different quality in the process of making flour products
}

\author{
Ang Zhang* \\ Puyang Vocational and Technical College, Puyang, Henan 457000, PR China
}

Received: 11 June 2020 / Accepted: 11 September 2020

\begin{abstract}
All kinds of flour products which are made of wheat flour have already become the staple food on people's dining table. With the improvement of living standard, the quality requirements for the flour products have gradually improved. In addition to the production technique, the quality of wheat flour as the raw material is also very important for the quality of flour products. This paper briefly introduced wheat flour and two main components that affected its quality: starch and protein. Then, the related quality of starch and protein and the quality of corresponding flour products were tested for five kinds of wheat flour. The experimental results showed that the quality indexes of five kinds of wheat flour were obviously different and representative. The regression analysis on the quality data of wheat flour and flour products showed that the water absorption of flour decreased with the increase of total starch content, dry gluten content and gluten index, the water solubility of flour decreased with the increase of total starch content and gluten index and increased with the increase of ratio of amylose to amylopectin, and the radial expansion rate of the flour products decreased with the increase of wet gluten content. To sum up, the quality of wheat flour will affect the water absorption, water solubility and radial expansion rate of flour products in the production process.
\end{abstract}

Keywords: Wheat flour / quality / flour products / production impact

\section{Introduction}

Flour products such as bread, steamed bread and noodles are made of wheat flour as the main food of human beings. The mature wheat becomes wheat flour after processing such as shelling and grinding. According to the fineness of grinding and the nutrients contained, wheat flour can be divided into different qualities [1]. After mixing wheat flour and water in a certain proportion, different kinds of flour products can be obtained, such as batter, dough, etc., and delicious food can be obtained after operations such as steaming or frying. With the improvement of living standards, the idea of eating gradually transforms from eating full, eating good to eating healthy [2]. For the flour products made from wheat flour, people have higher and higher quality requirements. Although the taste and nutrition of the flour products made from wheat flour partly depend on the processing level (including artificial and mechanical), more importantly, it depends on the quality of wheat flour as the raw material. The proportion of various nutrients in wheat flour of different quality is very different, which will affect the final product quality

\footnotetext{
* Corresponding author: angz_za@yeah.net
}

when making flour products [3]. The most simple and intuitive example is that different kinds of wheat flour will be selected when making different kinds of flour products in daily life. In order to improve the quality of flour products, it is necessary to study wheat flour of different quality. In the study of Mi et al. [4], they analyzed the quality changes of immature wheat in 24 weeks under different storage temperatures and established the Arrhenius model to predict the quality changes. The experimental results showed that the volatile compounds content of immature wheat significantly increased under the relatively high temperature storage environment of $35-45^{\circ} \mathrm{C}$ and the cookies made from this material were more popular. $\mathrm{Yu}$ et al. [5] reduced the content of deoxynivalenol in wheat flour using ozone and ultraviolet, so as to treat the contaminated wheat flour and found that both methods could effectively reduce the content of deoxynivalenol in wheat flour and had no effect on the protein, wet gluten content and whiteness of wheat flour. Narwal et al. [6] studied the quality change of wheat flour after adding different proportion of barley flour and found through experiments that the addition of barley flour could significantly improve the content of $\gamma$-glucan and antioxidant activity of wheat flour and its products. This paper briefly introduced the wheat flour and two main 
components that affected its quality: starch and protein. Then, the related quality detection of starch and protein and the quality detection of corresponding flour products were carried out for five kinds of wheat flour. The determination methods are as follows. The acid hydrolysis method was used for determining the total starch content of wheat flour, the iodine blue colorimetric method for determining the amylose content, the Kjeldahl determination method for determining the protein content in wheat flour, and the gluten meter for determining the gluten content of wheat flour. After wheat flour processing, the vacuum freeze-drying method was used for determining the water absorption and water solubility of flour products, the oil immersion method for determining the holding capacity of flour products, and the vernier caliper for determining the radial expansion rate of flour products.

\section{Wheat flour}

Wheat grains can be divided into embryo, endosperm and seed coat structurally. The main components of wheat are concentrated in endosperm, which contains starch and protein [7]. When wheat is processed into wheat flour, its seed coat and embryo will be removed as much as possible, and only the endosperm will be grinded into flour, that is to say, wheat flour is composed of wheat endosperm. Therefore, the main components of wheat flour are starch and protein, which are the main factors affecting the quality of wheat flour. The quality of wheat flour is usually determined by the content of starch and protein.

Generally, the starch content of dry wheat flour accounts for three quarters of its total weight, which is the most abundant component in dry wheat flour. Starch is a general term for a kind of polysaccharide complex. Its spatial structure is mostly chain or branched chain with different length. When it enters the digestive system of human body, it decomposes into glucose under the action of enzyme and is absorbed by human body for energy consumption [8]. When wheat flour is used to make bread, steamed bun and steamed bread, yeast will be added to make the dough expand to improve the taste quality. In the process of dough fermentation, the damaged starch in wheat flour is more likely to be broken down into different sugars by yeast and further improve the number of yeast. In this process, in addition to the increase of sugars, carbon dioxide will be generated. These gases will be confined by the swelling starch and gluten after water absorption and finally make the dough expand. After baking or steaming, the products will have a fluffy taste. In addition to the damaged starch, there are amylose and amylopectin in the starch of wheat flour. When wheat flour is made into flour products, the gelatinized amylopectin will gradually age, which will affect the quality of flour products.

Protein is the second most important component in wheat flour, which mainly exists in the endosperm of wheat grains. The higher the processing accuracy of wheat flour, the more protein loss it will have [9]. When wheat flour and water are mixed in a certain proportion, dough will be formed. The reason is that the gluten network formed by the above-mentioned wheat gliadin and gluten after adding water and the gluten network will wrap the starch particles after absorbing water. In the process of dough kneading, the protein in the gluten network repeats the process of polymerization and depolymerization, the covalent bond for combination gradually increases, and the gluten network gradually becomes strong. However, if the kneading time is too long, the number of covalent bond breaks will increase, and the quality of dough will decline. As mentioned above, yeast will be added to wheat flour when making bread, bun and other flour products. In the process of dough kneading, the enzyme produced by yeast will not only decompose starch to produce carbon dioxide, but also decompose protein in dough to produce amino acids, so as to improve the nutritional value of flour products. In addition, polypeptides and amino acids produced by protein decomposition will make flour products have a unique flavor.

\section{Experimental analysis}

\subsection{Experimental materials}

Experimental materials: No.1 flour: Fulinmen general wheat flour with wheat core; No. 2 flour: Xuehua wheat flour; No.3 flour: Golden dragon fish cake flour; No. 4 flour: Xiangmanyuan wheat flour; No. 5 flour: Wudeli Jinte refined high gluten flour.

Test reagents: edible salt, monoglyceride, distilled water, hydrochloric acid, $\mathrm{NaOH}$ solution, ether, ethanol, iodine solution, concentrated sulfuric acid, potassium permanganate solution, and vegetable oil.

Experimental instruments (Fig. 1): extrusion-expansion machine, chalking machine, spectrophotometer, automatic nitrogen determinator, gluten testing system, high-speed centrifugal, and vernier caliper.

\subsection{Production of wheat flour products}

The production process of wheat flour products used in this experiment is shown in Figure 2. First, the materials needed for making flour products were weighted, including $10 \mathrm{~kg}$ of wheat flour, $1 \mathrm{~kg}$ of edible salt, $48 \mathrm{~g}$ of monoglyceride and $2.4 \mathrm{~kg}$ of water; then they were dissolved and mixed tin water; the mixture obtained after initial dissolution was poured into the powder beater for full mixing; the dough that has been mixed evenly was moved to the extrusion-expansion machine after $15 \mathrm{~min}$; the flour product was obtained after operating according to the operation manual.

\subsection{Experiment item}

(1) Measurement of total starch in wheat flour: the content of total starch in the wheat flour was detected by acid hydrolysis method [10]. Firstly, $5 \mathrm{~g}$ of wheat flour was taken, and the fat and soluble sugar were removed with ether and ethanol; then the wheat flour was hydrolyzed with $6 \mathrm{~mol} / \mathrm{L}$ hydrochloric acid; the hydrolysate was neutralized with $\mathrm{NaOH}$ solution after hydrolysis; the hydrolysate was diluted with a $500 \mathrm{ml}$ volumetric flask; 


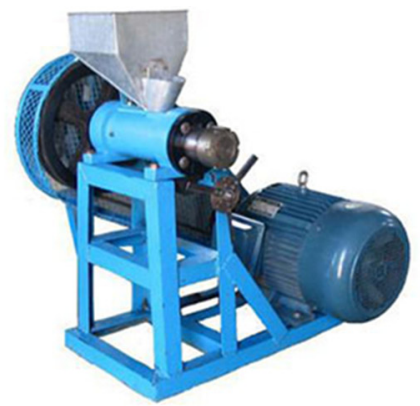

Extrusion-expansion machine

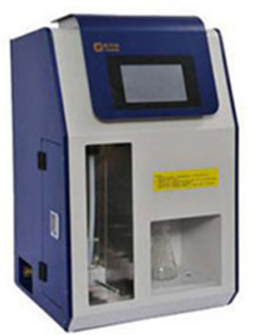

Automatic nitrogen determinator

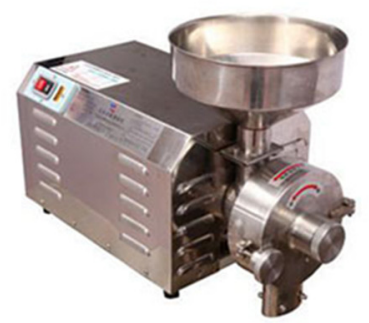

Chalking machine

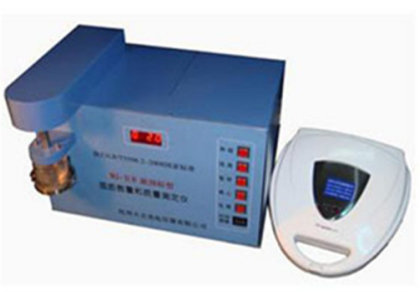

Gluten testing system

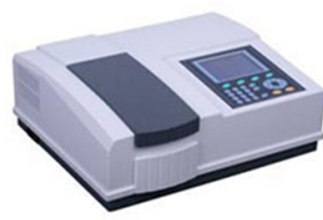

Spectrophotometer

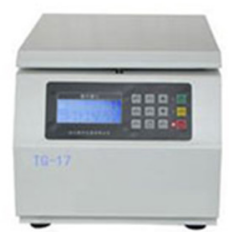

High-speed centrifugal

Fig. 1. The instruments for experiments.

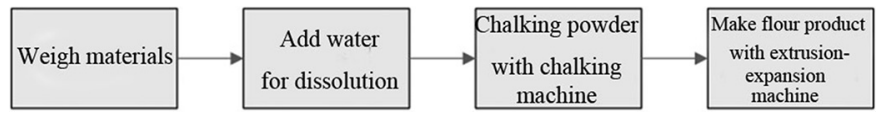

Fig. 2. Production process of wheat flour products.

sample was taken from the volumetric flask, and the content of reducing sugar in the sample was determined by potassium permanganate method; the starch content in the wheat flour was calculated by the formula as follows:

$$
\text { Content of } \operatorname{starch}=\frac{0.9 \mathrm{~m}}{10 \mathrm{~V}} \times 100 \%,
$$

where $m$ is the content of reducing sugar measured in the sample from the volumetric flask, unit: $\mathrm{mg}$, and $V$ is the volume of the sample from the volumetric flask, unit: $\mathrm{mL}$.

(2) Measurement of amylose in wheat flour: the content of amylose in the wheat flour was detected by iodine blue colorimetry [11]. Firstly, the concentration standard curve of amylose was drawn by using five different concentrations of amylose standard solution. Then some wheat flour was taken and processed by alcohol, $\mathrm{NaOH}$ solution dispersion and water bath. The absorbance was detected using $620 \mathrm{~nm}$ spectrophotometer after adding distilled water, acetic acid and iodine solution. The content of amylose in the wheat flour was obtained by referring to the standard curve.

(3) Measurement of total protein of wheat flour: the total protein of the wheat flour was detected by kjeldahl method [12] with an automatic nitrogen determinator. First, $5.0 \mathrm{~g}$ of wheat flour sample was taken and put into the automatic nitrogen determinator. The temperature was controlled by program, keeping at $280^{\circ} \mathrm{C}$ for $30 \mathrm{~min}$ and $430^{\circ} \mathrm{C}$ for $90 \mathrm{~min}$. After digestion and cooling, the automatic nitrogen determinator automatically carried out titration detection to obtain the total protein content of the wheat flour.

(4) Measurement of gluten in wheat flour: the content of gluten in the wheat flour was detected using a gluten tester. Firstly, some sample was taken and made to absorb $2 \%$ salt water. Then the sample was washed using the gluten tester until the gluten was obtained, and the obtained gluten was screened. The non-screened gluten was wet gluten; after drying, it became dry gluten. The weight of the wet gluten and dry gluten was weighted to obtain the content of wet and dry gluten and gluten index of the sample [13].

(5) Measurement of water absorption and water solubility of flour products: after the sample of the flour product was dried by vacuum freeze-drying to remove water, it was crushed into powder and weighed. It was mixed evenly with distilled water in a centrifuge tube. After a period of water bath, it was centrifuged in a centrifuge to weigh the mass of precipitate and supernatant respectively after drying. The calculation formulas of water absorption and water solubility [14] are as follows:

$$
\left\{\begin{array}{l}
\mathrm{WAI}=\frac{M_{5}-M_{2}}{M_{1}} \times 100 \% \\
\mathrm{WSI}=\frac{M_{4}-M_{3}}{M_{1}} \times 100 \%
\end{array}\right.
$$


Table 1. Quality index analysis of starch and protein in wheat flour.

\begin{tabular}{llllllll}
\hline Project index & $\begin{array}{l}\text { Total } \\
\text { starch } \\
\text { content\% }\end{array}$ & $\begin{array}{l}\text { Amylose } \\
\text { content\% }\end{array}$ & $\begin{array}{l}\text { Ratio of } \\
\text { amylose to } \\
\text { amylopectin }\end{array}$ & $\begin{array}{l}\text { Total } \\
\text { protein } \\
\text { content\% }\end{array}$ & $\begin{array}{l}\text { Wet } \\
\text { gluten } \\
\text { content\% }\end{array}$ & $\begin{array}{l}\text { Dry } \\
\text { gluten } \\
\text { content\% }\end{array}$ & $\begin{array}{l}\text { Gluten } \\
\text { Index }\end{array}$ \\
\hline No. 1 flour & 70.5 & 10.5 & 0.18 & 9.9 & 28.4 & 5.2 & 59 \\
No. 2 flour & 72.3 & 12.3 & 0.21 & 10 & 30.2 & 7.3 & 66 \\
No. 3 flour & 74.5 & 13.4 & 0.22 & 10.3 & 41.5 & 5.6 & 73 \\
No. 4 flour & 75.6 & 11.2 & 0.17 & 11.5 & 29.8 & 8.3 & 84 \\
No. 5 flour & 77.1 & 14.4 & 0.23 & 12.3 & 43.9 & 10.4 & 92 \\
Average value & 74 & 12.36 & 0.20 & 10.8 & 34.76 & 7.36 & 74.8 \\
Coefficient of variation & 3.88 & 10.56 & 15.33 & 8.25 & 16.44 & 26.58 & 16.55 \\
\hline
\end{tabular}

Table 2. Regression analysis of factors influencing the quality index of flour products.

\begin{tabular}{|c|c|c|c|c|c|c|c|c|}
\hline \multirow[b]{2}{*}{ Variable } & \multicolumn{2}{|c|}{$\begin{array}{l}\text { Water absorption } \\
\text { index } \%\end{array}$} & \multicolumn{2}{|c|}{$\begin{array}{l}\text { Water solubility } \\
\text { index\% } \\
\end{array}$} & \multicolumn{2}{|c|}{$\begin{array}{l}\text { Oil holding } \\
\text { capacity\% }\end{array}$} & \multicolumn{2}{|c|}{$\begin{array}{l}\text { Radial expansion } \\
\text { rate\% } \\
\end{array}$} \\
\hline & $k$ & $P$ value & $k$ & $P$ value & $k$ & $P$ value & $k$ & $P$ value \\
\hline Total starch content $\%$ & -0.817 & 0.047 & -0.911 & 0.041 & -0.296 & 0.151 & 0.041 & 0.211 \\
\hline Amylose content $\%$ & 0.256 & 0.125 & 0.784 & 0.256 & -0.384 & 0.131 & -0.613 & 0.321 \\
\hline $\begin{array}{l}\text { Ratio of amylose } \\
\text { to amylopectin }\end{array}$ & 0.593 & 0.245 & 0.935 & 0.003 & -0.213 & 0.147 & -0.423 & 0.154 \\
\hline Total protein content $\%$ & -0.603 & 0.251 & 0.036 & 0.222 & -0.032 & 0.216 & -0.789 & 0.325 \\
\hline Wet gluten content $\%$ & -0.265 & 0.145 & 0.143 & 0.321 & -0.412 & 0.214 & -1.011 & 0.002 \\
\hline Dry gluten content\% & -0.831 & 0.036 & -0.312 & 0.123 & -0.333 & 0.156 & -0.741 & 0.154 \\
\hline Gluten index & -0.956 & 0.005 & -0.874 & 0.044 & -0.134 & 0.222 & -0.231 & 0.321 \\
\hline Constant term & 491.36 & - & 12.1 & - & 30.12 & - & 14.21 & - \\
\hline
\end{tabular}

where WAI and WSI stand for water absorption index and water solubility index, $M_{1}$ is the mass of the sample used for testing, $M_{2}$ is the mass of centrifugal pipe, $M_{3}$ is the mass of aluminum box, $M_{4}$ is the total mass of supernatant and aluminum box after drying, and $M_{5}$ is the total mass of the centrifuged sediment and the centrifuge tube.

(6) Measurement of oil holding capacity of flour product: some flour product sample was taken and soaked in vegetable oil for half an hour. Then it was taken out, and the vegetable oil on the surface of the sample was absorbed using filter paper. It was centrifugated in a centrifuge for a period of time, and the vegetable oil on the surface of the sample was absorbed again. The mass of the sample was weighted. The calculation formula of oil holding capacity is:

$$
\text { Oil holding capacity }=\frac{W_{2}-W_{1}}{W_{1}} \times 100 \%,
$$

where $W_{1}$ is the initial mass of the sample and $W_{2}$ is the mass of the sample after immersion in oil and centrifugation.

(7) Measurement of radial expansion rate of flour product [15]: the diameter of the flour product and the diameter of mould outlet were measured using vernier caliper respectively, so as to calculate the radial expansion rate of the flour product.
The above experiments were repeated for 5 times for each kind of wheat flour and corresponding flour products, and the average result was taken as the final result.

\subsection{Experimental results}

As shown in Table 1, the coefficient of variation represents the fluctuation of the difference between the data under the corresponding quality index (the greater the fluctuation, the greater the data fluctuation), reflecting the quality difference between different varieties of wheat flour and the quality difference of flour products made from different wheat flour. It was seen from the data in Table 1 that there were obvious differences between the five wheat flours, and the variation coefficients of amylose content, dry and wet gluten content and gluten index were relatively large, which indicated that the above quality indexes of the five wheat flours were quite different and further indicated that the five wheat flours were representative in quality.

As shown in Table 2, $k$ represents the standardized regression coefficient of the corresponding variable, and $\mathrm{P}$ value represents whether the regression coefficient of the variable is significantly related to the variable, smaller than 0.05 as significantly related. It was seen from Table 2 that water absorption index $=491.36-0.817 \times$ total starch content $-0.835 \times$ dry gluten content $-0.956 \times$ gluten index; water solubility index $=12.1-0.911 \times$ total starch 
content $+0.935 \times$ amylopectin ratio $-0.874 \times$ gluten index; radial expansion rate $=14.21-1.011 \times$ wet gluten content. According to the regression equation obtained from the above regression analysis, it was seen that the factors influencing the water absorption index of wheat flour were total starch content, dry gluten content and gluten index, all of which had negative effects on the water absorption index, and gluten index was the most influential factor, which was consistent with the above correlation analysis; the factors influencing the water solubility index of wheat flour were total starch content, ratio of amylose to amylopectin and gluten index, total starch content and gluten index had negative effects on water solubility index, ratio of amylose to amylopectin had positive effects, and ratio of amylopectin had the greatest impact, which was consistent with the above correlation analysis; the wheat flour quality variable factor of radial expansion rate of flour products was wet gluten content, and it had negative effects on radial expansion rate, which was consistent with the above correlation analysis.

\section{Discussion}

The correlation analysis showed that the water absorption index of flour products was negatively correlated with the total starch content, dry gluten content and gluten index of wheat flour. The equation obtained after regression analysis was: water absorption index $=491.36-0.817 \times$ total starch content $-0.835 \times$ dry gluten content $0.956 \times$ gluten index, which was also consistent with the correlation analysis. It was seen from the equation that the water absorption of the flour products made of wheat flour would reduce with the increase of total starch content, dry gluten content and gluten index. The reason might be that the increase of gluten protein made the flour products form a closer gluten network in the process of making, thus occupying the space of other ingredients, or wrapping the ingredients with hydrophilic groups more tightly, which eventually made the ingredients of the dough difficult to bond with water molecules.

The water solubility index of flour products had obvious negative correlation with the total starch content and gluten index of wheat flour, but had more obvious positive correlation with the ratio of amylose to amylopectin. The equation obtained by regression analysis was: water solubility index $=12.1-0.911 \times$ total starch content + $0.935 \times$ ratio of amylose to amylopectin $-0.874 \times$ gluten index, which was also consistent with the correlation analysis. It was from the regression equation that with the increase of total starch content and gluten index in the flour, the water solubility of flour products made from wheat flour would decrease, but it would increase with the increase of the ratio of amylose to amylopectin. The reason might be that the increase of the ratio of amylose to amylopectin meant the increase of amylose content, the mixing and kneading of wheat flour dough and the extrusion at the outlet of the extruder would make the amylose break in the process of making flour products, and soluble substance produced in that process would produce to make it easier to combine with water.
There was a significant negative correlation between the radial expansion rate of flour products and the wet gluten content of wheat flour. The equation obtained by regression analysis was: the radial expansion rate $=14.21-1.011 \times$ wet gluten content, which was also consistent with the correlation analysis. It was seen from the equation that the radial expansion rate of flour products made of wheat flour would be reduced with the increase of the wet gluten content of wheat flour. The reason might be that wet gluten which produced due to the water absorption of some gluten protein in wheat flour would make dough combine tighter and the water loss caused by the compression of mould outlet on the dough would be less if there was higher content of wet gluten.

The above methods can effectively determine the difference of the composition content of wheat flour with different quality and the quality of flour products made from corresponding wheat flours. However, the limitation of this method is that although all the indexes tested reflected some characteristics of the test subject, they could not reflect characteristics more comprehensively. Moreover, more samples are needed to determine the exact relationship between wheat flour quality and flour product quality, which is also the future research direction.

\section{Conclusion}

In this paper, the wheat flour and the starch and protein contained were introduced briefly. Then five types of wheat flour were selected and their quality was tested, including total starch content, amylose content, ratio of amylose to amylopectin, total protein content, dry and wet gluten content and gluten index. Then the wheat flour was processed into flour products by extrusion extruder, and the relevant quality was detected, including water absorption index, water solubility index, oil holding capacity and radial expansion rate. Finally, the correlation analysis of quality index was carried out. The results are as follows: (1) the variation coefficients of quality indexes of wheat flours and flour products were large, and the quality differences between different kinds of wheat flours and flour products were large, which was representative; (2) the water absorption index of flour products was negatively correlated with the total starch content, dry gluten content and gluten index of wheat flour, among which the gluten index was more correlated; the water solubility index of flour products is negatively correlated with the total starch content and gluten index of wheat flour and more positively correlated with the ratio of amylose to amylopectin; the oil holding capacity was not significantly correlated with the quality indexes of wheat flour; there was a significant negative correlation between the radial expansion rate and the wet gluten content of wheat flour; (3) the regression equation between the quality of flour products and the quality of wheat flour was: water absorption index $=491.36-0.817 \times$ total starch content $-0.835 \times$ dry gluten content $-0.956 \times$ gluten index; water solubility index $=12.1-0.911 \times$ total starch content $+0.935 \times$ ratio of amylose to amylopectin $-0.874 \times$ gluten index; radial expansion rate $=14.21-1.011 \times$ wet gluten content. 


\section{References}

1. T. Liu, G.G. Hou, M. Cardin, L.F. Marquart, A. Dubat, LWT - Food Sci. Technol. 77, 1-7 (2017)

2. S. Ketjarut, R. Pongsawatmanit, Int. J. Food Eng. 11, 641-650 (2015)

3. T. Bojňanská, J. Šmitalová, A. Vollmannova, M. Tokár, V. Vietoris, J. Microbiol. Biotechnol. Food Sci. 4, 18-22 (2015)

4. M.J. Kim, H.S. Kwak, M.J. Lee, S. Kim, LWT - Food Sci. Technol. 83, 42-49 (2017)

5. Y.G. Yu, H.R. Ma, R. Hou, Y.Q. Tang, X.L. Xiao, Mod. Food Sci. Technol. 32, 196-202 (2016)

6. S. Narwal, D. Kumar, S. Sheoran, R.P.S. Verma, R.K. Gupta, J. Food Sci. Technol. 54, 1-7 (2017)

7. S. Wang, A. Opassathavorn, F.J. Zhu, Texture Stud. 46, 281-292 (2015)
8. M. Hruskova, R. Varacek, Czech J. Food Sci. - UZPI (Czech Republic) 19, 189-195 (2018)

9. D.L. Zhang, T.T. He, H.H. Liang, L.Y. Huang, Y.Z. Su, Y.G. Li, S.P. Li, J. Agric. Food Chem. 64, 5128-5136 (2016)

10. N.Y. Lee, Prev. Nutr. Food Sci. 21, 361-366 (2016)

11. M.S. Coelho, M. Salas-Mellado, LWT - Food Sci. Technol. 60, 729-736 (2014)

12. H. Tanaka, C. Nabeuchi, M. Kurogaki, M. Garg, M. Saito, G. Ishikawa, T. Nakamura, H. Tsujimoto, Breeding Sci. 67, 509-517 (2017)

13. O.F. Ekunseitan, A.O. Obadina, O.P. Sobukola, A.M. Omemu, M.O. Adegunwa, O.E. Kajihausa, A.A. Adebowale, S.A. Sanni, L.O. Sanni, K. Tomlins, J. Food Process. Pres. 41 (2016)

14. V. Giannou, C. Tzia, Foods 5, 6 (2016)

15. A. Carrubba, A. Comparato, A. Labruzzo, S. Muccilli, V. Giannone, A. Spina, J. Food Sci. 81, C2158-C2166 (2016)

Cite this article as: Ang Zhang, Effect of wheat flour with different quality in the process of making flour products, Int. J. Metrol. Qual. Eng. 11, 6 (2020) 